\title{
Produção de crisântemo (Dendranthema grandiflora Tzvelev.) 'Snowdon' em vaso II: ciclo da cultivar, comprimento, largura e área da folha
}

\author{
Production of chrysanthemum (Dendranthema grandiflora Tzvelev.) 'Snowdon' in pot II: cycle, leaf \\ length, width and area
}

\author{
Jucelma de Cássia C. Tolotti Mainardi ${ }^{1}$ Rogério Antônio Bellé ${ }^{2}$ Leandro Mainardi ${ }^{3}$
}

\section{RESUMO}

O objetivo do trabalho foi avaliar a resposta quanto ao ciclo e comprimento, largura e área da folha, ao redutor de crescimento Daminozide, pulverizado na cultivar de crisântemo (Dendranthema grandiflora Tzvelev.) de corte Snowdon, conduzida em vaso. $O$ estudo foi composto por dois ensaios com cinco repetições, no delineamento experimental inteiramente casualizado bifatorial $4 \times 2$, sendo formado por quatro doses de Daminozide (0, 2000, 4000 e 6000mg. L $\left.^{-1}\right)$ e duas freqüencias de aplicações (semanal e bissemanal). Os resultados mostraram que a cultivar Snowdon pode ser conduzida em vaso, porém, com aumento de ciclo e redução de até $28 \%$ na área da folha.

Palavras-chave: redutor de crescimento, daminozide (ácido succínico-2,2-dimetilhidrazida), B-nine, dose, freqüência de aplicação.

\section{ABSTRACT}

The aim of this study was to investigate the response of cultivation cycle, length, width and area leaf, to the growth retardant Daminozide, sprayed on the chrysanthemum (Dendranthema grandiflora Tzvelev.) 'Snowdon' cultivated in pot. The study consisted of two experiments with five repetitions. The experiments were bifactorial $4 \times 2$, with four concentrations of Daminozide (0, 2.000, 4.000 and 6.000 $\left.\mathrm{mg}^{. \mathrm{L}^{-1}}\right)$ and two frequencies of application (weekly and bi-weekly) of the product. Cultivation cycle, leaf length, width and area were determined. The 'Snowdon' was able to produce pots commercially acceptable, with increase in the cultivation cycle and decrease of the leaf size.

Key words: growth retardant, succinic acid 2-2-dimethylhydrazide (daminozide), B-nine, concentration, frequency of application.

\section{INTRODUÇÃO}

O crisântemo de vaso é uma das principais flores produzidas em estufas e com constante aumento de produção. Isso ocorre devido à cultura apresentar principalmente grande diversidade de formas e cores das inflorescências, precisão da resposta ao fotoperíodo e longevidade das inflorescências.

No Rio Grande do Sul, a principal espécie de vaso cultivada é o crisântemo, cuja produção é insuficiente para atender o mercado. Uma das causas dessa deficiência é devido às limitações térmicas e de radiação, especialmente no inverno, o que dificulta a produção contínua. Embora o mercado apresente um grande número de cultivares de vaso, essas ainda não o satisfazem quanto a adaptabilidade para o cultivo em regiões de invernos frios, especialmente as do tipo bola. Segundo BELLÉ (1998), as cultivares do tipo bola de corte podem ser conduzidas em vaso, desde que, o manejo dos redutores de crescimento seja equacionado.

As cultivares de corte tipo bola 'Snowdon' e 'Yellow Snowdon' não apresentam limitações térmicas na produção durante o ano todo no Rio Grande do Sul. Por essa razão, seu cultivo em vaso pode se tornar um produto diferenciado, permitindo uma melhor comercialização.

A cultivar Snowdon é classificada como: tipo encurvado ou bola, ciclo longo ( 9 semanas) e cor

\footnotetext{
${ }^{1}$ Engenheiro Agrônomo, Doutorando em Agronomia pela Universidade Federal de Santa Maria (UFSM). E-mail: lemain@terra.com.br. Autor para correspondência.

${ }^{2}$ Engenheiro Agrônomo, Doutor, Professor Adjunto do Departamento de Fitotecnia, UFSM, 97105-900-Santa Maria, RS. E-mail: bellé@ccr.ufsm.br.

${ }^{3}$ Engenheiro Agrônomo, Especialista em Administração e Desenvolvimento Rural, Autônomo, Santa Maria, RS.
} 
branca. É utilizada para corte, possui inflorescência grande, hastes longas e alto vigor. Foi especialmente melhorada para ser conduzida com uma única inflorescência, cujo diâmetro pode chegar a mais de 20 cm (SCHOENMAKER, 2000).

Mesmo em cultivares de porte baixo, especialmente melhoradas para cultivo em vaso, o controle da altura através de redutores de crescimento faz-se necessário para adequar a estética do seu porte em relação ao tamanho do vaso.

O tratamento com esses redutores é feito durante a fase de rápido crescimento vegetativo ou mesmo logo após o início do crescimento do botão, quando as plantas estão no tamanho limite da altura (HERTWIG, 1977 e HARTMANN et al., 1988).

Os retardadores de crescimento atuam dentro da planta inibindo a produção natural de giberelina, o que modifica a morfologia da planta, com redução do seu tamanho. Eles afetam a formação de células e a elongação do entrenó abaixo do meristema. Assim, são obtidas plantas pequenas com o desenvolvimento de flores normais. O comprimento do entrenó é reduzido, contudo o número de entrenós normalmente não é afetado. Além disso, as folhas são menores e ficam com um verde mais intenso (BARRET, 1992).

Especificamente sobre o redutor de crescimento Daminozide (ácido succínico-2,2dimetilhidrazida), conhecido comercialmente como Sadh, Alar-85, B-nine e Kilar, ainda segundo BARRET (1992), o produto é aplicado somente via foliar sendo muito móvel em todas as partes da planta. Sua concentração em pulverização está compreendida entre 1.250 a $5.000 \mathrm{mg} . \mathrm{L}^{-1}$ e seu efeito depende de alguns fatores como: idade da planta e temperatura.

O mecanismo de ação desse fitohormônio ainda não é bem conhecido. Segundo LOZOYA-SALDAÑA (1992) ele inibe o ácido indolacético. A resposta fisiológica dos tecidos sob ação dessa auxina é ampla, destacando-se pelo efeito de elongação, divisão e diferenciação celular (PENON, 1982). Já ARTECA (1995) sugere que o Daminozide pode afetar a biossíntese de giberelina.

De acordo com LOPES (1977), para o crisântemo, a freqüência e dosagem das aplicações de Daminozide dependem ainda da cultivar, da estação do ano, do tamanho da planta e do vaso. O produto deve ser aplicado de uma a quatro vezes, em média, durante o ciclo, utilizando-se de 2.000 a 3.000mg.L $\mathrm{L}^{-1}$. Mas, há casos em que são necessárias mais de quatro aplicações para que o vaso chegue ao mercado com o porte ideal.

Estudos conduzidos por TAYAMA (1992) mostraram que, para cultivares muito sensíveis, os melhores efeitos do Daminozide foram obtidos com a concentração de $0,25 \%$ (2.500mg. $\left.\mathrm{L}^{-1}\right)$, aplicado quando as plantas atingiram a altura final desejada. Para cultivares sensíveis ou pouco sensíveis a melhor concentração foi de $0,5 \%\left(5.000 \mathrm{mg} . \mathrm{L}^{-1}\right)$, pulverizando o produto quando as brotações laterais estavam com quatro a cinco centímetros de comprimento. Entretanto, aplicações posteriores podem ser necessárias após duas semanas. Se estas forem realizadas quatro semanas antes do período de florescimento, poderão afetar a coloração das flores, principalmente em cultivares brancas.

Os redutores de crescimento podem ter uma ação retardadora sobre o ciclo de desenvolvimento do crisântemo. Além disso, em condições de estufa, também pode ocorrer atraso no ciclo, como foi constatado por NARDI (2000) que para o período correspondente de março a novembro, foi possível o cultivo de corte da cultivar Snowdon nas condições de Santa Maria - RS, porém, com aumento de ciclo de uma semana que deve ser considerado na programação da produção comercial.

Em plantas com botões florais já iniciados, o tratamento com Daminozide estudado por CATHEY (1975), geralmente altera o desenvolvimento do eixo floral e atrasa o florescimento sem retardar significativamente a elongação da haste.

A aplicação de Daminozide na concentração de 5.000mg. $\mathrm{L}^{-1}$, em dezesseis cultivares tardias de crisântemo de vaso, foi pesquisada por WEI \& BIWEN (1997) e verificaram que o produto retardou o florescimento, mas sugeriram que isso poderia ser neutralizado pulverizando os brotos com uma solução de somente $5 \mathrm{mg} . \mathrm{L}^{-1} \mathrm{GA}_{3}$.

No entanto, trabalhos desenvolvidos por YONGKWEON et al. (1999) indicam que o Daminozide aplicado em Chrysanthemum zawadskii ssp. naktongense, não teve efeito sobre os dias para o florescimento. Também EL-KELTAWI et al. (1996), estudando o crescimento de Chrysanthemum, usando Alar (Daminozide na concentração de 0, 500 e $\left.1.000 \mathrm{mg} . \mathrm{L}^{-1}\right)$ mostraram que o produto não teve efeito sobre as características do florescimento.

Os efeitos dos redutores de crescimento também podem ser observados sobre o tamanho, cor das folhas e pedúnculos foliares da planta. Esses parâmetros são importantes para a qualidade, pois o fato dos redutores produzirem plantas menores, pela aproximação das folhas, deixa o vaso mais compacto e com melhor estética. Entretanto, há uma redução do tamanho das mesmas que pode descompensar o efeito estético em relação à cobertura foliar do vaso.

Em pesquisa realizada por CATHEY (1975), o crisântemo respondeu ao retardador químico de crescimento pela lenta elongação da haste/caule e desenvolveu folhas com tonalidade verde escuro, mas o produto promoveu o esverdeamento intenso somente nas 
folhas imaturas, com pouca intensificação do verde nas folhas mais velhas. Folhas afetadas não se expandiram por completo, mas apresentaram espessamento da camada do parênquima paliçádico.

Os efeitos do tratamento em pulverização com Daminozide e do desponte, sobre o crescimento e florescimento de Chrysanthemum zawadskii ssp. naktongense foram investigados por YONGKWEON et al. (1999). Eles também verificaram que as folhas apresentavam tamanho reduzido e a concentração de 50 $\mathrm{mg} /$ vaso diminuiu o comprimento e a largura da folha.

O presente trabalho teve como objetivo avaliar a resposta da cultivar de crisântemo Snowdon a diferentes doses e frequiências de aplicação do redutor de crescimento Daminozide quanto ao ciclo, comprimento, largura e área da folha.

\section{MATERIAL E MÉTODOS}

Foram conduzidos dois ensaios em casa de vegetação, pertencente ao Setor de Floricultura, do Departamento de Fitotecnia, da Universidade Federal de Santa Maria, RS, Brasil (latitude $29^{\circ} 43^{\prime} \mathrm{S}$, longitude $53^{\circ}$ 42'W e altitude $95 \mathrm{~m}$ ).

O enraizamento das estacas foi realizado em vaso no 15 com três mudas cada. Considerou-se o tempo de duas semanas como findo o processo de enraizamento. A partir desse momento, dois ensaios foram conduzidos, um com mais uma semana de dias longos (DL) e o outro com mais duas semanas de DL. Cada um desses foi conduzido em esquema bifatorial $4 \times 2$ no delineamento inteiramente casualizado, com cinco repetições. $\mathrm{O}$ primeiro fator foi composto por quatro doses $\left(0,2.000,4.000\right.$ e $\left.6.000 \mathrm{mg}^{-\mathrm{L}^{-1}}\right)$ do redutor químico de crescimento Daminozide, quimicamente puro (ácido succínico - 2, 2 dimetilhidrazida). $\mathrm{O}$ outro fator foi constituído por duas freqüências de aplicação, semanal (7 em 7 dias) e bissemanal (14 em 14 dias), com o uso da cultivar Snowdon.

O início do enraizamento deu-se em 24/03/99 em câmara úmida por 14 dias sob condições de DL. Considerando-se esse tempo como término do enraizamento, um ensaio permaneceu em dia longo por mais uma semana (até 14/04/99) e o outro ensaio por mais duas semanas (até 21/04/99). Nessas datas, os vasos foram espaçados para 9 vasos.m² (MOTOS, 1998) e iniciaramse as pulverizações com o Daminozide ao mesmo tempo em que ocorreu a indução floral. As aplicações do produto finalizaram com botões de $1,5 \mathrm{~cm}$ de diâmetro, totalizando nove aplicações semanais e cinco bissemanais para o ensaio com uma semana de DL e oito aplicações semanais e quatro bissemanais para o ensaio com duas semanas de DL.

A adubação foi utilizada com a proporção $\mathrm{N}$ P K de 2:0,3:1 até o surgimento dos botões florais para formação das hastes, massa foliar e raízes. Após essa fase, a proporção N P K foi de 1,0:0,3:2,0.

Foram avaliados o ciclo, o comprimento $(\mathrm{cm})$, a largura $(\mathrm{cm})$ e a área da folha $\left(\mathrm{cm}^{2}\right)$.

$\mathrm{O}$ ciclo foi determinado pelo número de dias desde a indução até o ponto de comercialização, ou seja, quando $50 \%$ das inflorescências estavam abertas.

Para avaliar a área da folha, utilizou-se o método descrito por HALLAIRE et al. (1970), C x L (comprimento $\mathrm{x}$ largura) que para maior precisão necessitou ser corrigida através do fator $\mathrm{K}$, segundo o método proposto por BARROS et al. (1973), sendo: $K=S / X$ onde: $S=$ somatório da área real (obtida em mesa digitalizadora); $\mathrm{X}=$ somatório da área da figura circunscrita (obtida pelo método C X L). $\mathrm{O}$ valor calculado do fator $\mathrm{K}$ para a cultivar Snowdon nas condições do ensaio foi de 0,44 .

Para análise estatística dos dados, foi utilizado o programa Software Científico (SOC), realizando-se análise da variância e regressão dos dados, em nível de $5 \%$ de significância.

\section{RESULTADOS E DISCUSSÃO}

A análise da variância dos dados do ciclo da cultivar mostrou interação significativa das diferentes doses e freqüências de aplicações nos dois ensaios, cujas curvas de regressão estão apresentadas na figura 1 .

Observa-se que, no ensaio com uma semana de DL (Figura 1A), o ciclo da cultivar aumentou com o aumento da freqüência e da dose. O maior ciclo observado foi na dose de $6000 \mathrm{mg} . \mathrm{L}^{-1}$, onde esse variou de quatro dias nas aplicações semanais e de seis dias na freqüência bissemanal, em relação às plantas não tratadas. Enquanto que, para o ensaio de duas semanas de DL (Figura 1B), só houve aumento de ciclo nas aplicações semanais, cujo maior aumento observado foi de três dias, também para a maior dose.

Ao comparar-se o ciclo das plantas testemunhas dos dois ensaios, observa-se que no primeiro (Figura 1A) este foi de 67 dias (9,56 semanas) e no segundo (Figura1B) foi de 72 dias (10,28 semanas). Esse prolongamento foi provavelmente devido a deficiências de temperatura e radiação solar nas duas primeiras semanas de indução como já foi observado por KOFRANEK (1992). Esse aumento de ciclo dessa cultivar em relação às informações do fornecedor de mudas (SCHOENMAKER, 2000) deve ser considerado na programação dos plantios.

As diferenças de ciclo verificadas entre os tratamentos dos ensaios podem ser explicadas pelo fato das plantas apresentarem uma diferença de estágio fenométrico a cada aplicação, uma vez que a indução foi efetuada com diferença de uma semana. A eficácia de 


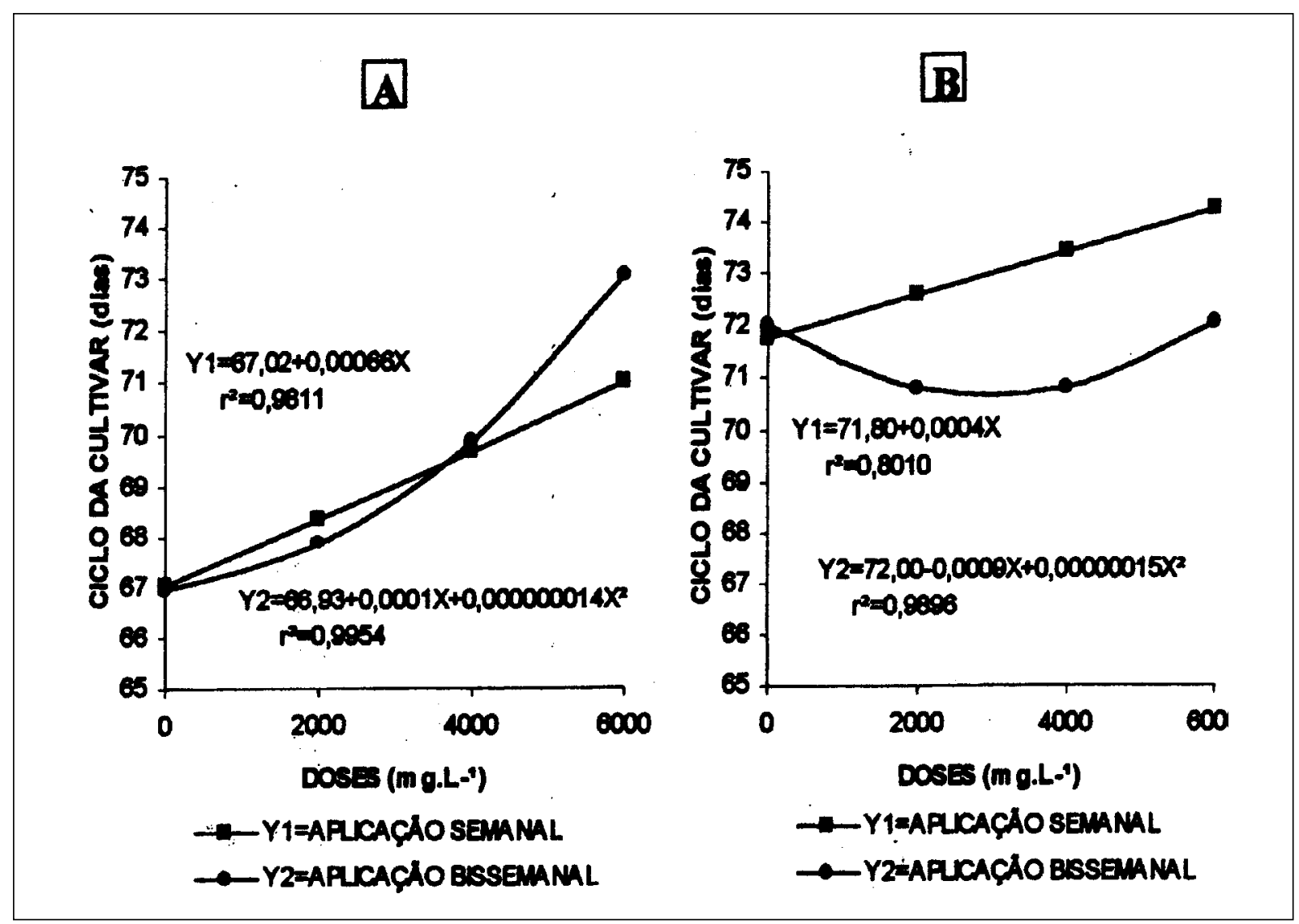

Figura 1 - Ciclo da cultivar Snowdon, para a interação doses x freqüências de Daminozide no ensaio com uma (A) e duas (B) semanas de DL. Santa Maria - RS, 2001.

ação do produto pode estar relacionada ao estágio, o que pode ter afetado diferentemente sua resposta.

Outro fator que pode ser atribuído ao prolongamento do ciclo com maior número de dias longos, pode ter sido o maior número de folhas formadas, em média duas folhas a mais, no ensaio com duas semanas de DL e isso pode ter determinado uma maior área de absorção do fitohormônio.

As aplicações do redutor Daminozide em doses elevadas apresentaram leve ação sobre a modificação do ciclo provavelmente pela não absorção pela planta do excesso da substância química (CATHEY, 1975), razão pela qual esse é pouco modificado.

O prolongamento de ciclo de diferentes espécies com o aumento de doses ou frequiências de aplicação pelo uso de Daminozide também foi observado por alguns autores: HARTLEY \& WILFRET (1992) com Poinsetia usando 2.000mg.L $\mathrm{L}^{-1} \mathrm{e}$ 3.000 mg.L. $\mathrm{L}^{-1} \mathrm{e}$ WEI \& BIWEN(1997) com crisântemo de vaso utilizando $5.000 \mathrm{mg} . \mathrm{L}^{-1}$.

Esse atraso na floração deve-se a inibição do ácido indolacético (LOZOYA-SALDAÑA, 1992), que é responsável pela ativação do processo de divisão e elongação celular, cujo bloqueio resulta em um atraso na diferenciação e crescimento das estruturas (PENON, 1982) e isso se repercute num atraso na abertura floral.

Quanto ao comprimento, à largura e à área da folha, a análise da variância dos dados mostrou que não ocorreu interação significativa entre as diferentes doses e frequiências de aplicação em ambos os períodos de DL.

Para essas variáveis, a freqüência de aplicação foi significativa somente no ensaio com duas semanas de DL, e sua comparação é mostrada na tabela 1. Nesta, observa-se que, na freqüência semanal, o comprimento, a largura e a área da folha foram significativamente menores que na freqüência bissemanal, indicando que, quando se diminuiu o tempo entre as aplicações, houve maior redução no tamanho da folha. As reduções verificadas foram de 7,98\% para o comprimento, $9,4 \%$ para a largura e $17,8 \%$ para a área da folha, independente da dose utilizada.

$\mathrm{O}$ efeito das doses sobre o comprimento da folha foi significativo para os dois ensaios analisados e observou-se (Figura 2A) uma redução no comprimento da folha. A maior redução foi de $14,60 \%$ verificada no

Ciência Rural, v.34, n.6, nov-dez, 2004. 
Tabela 1 - Comprimento, largura e área da folha, para diferentes frequiências de aplicações de Daminozide em crisântemo (Dendranthema grandiflora Tzvelev.), cultivar Snowdon, no ensaio com duas semanas de dias longos (DL). Santa Maria - RS, 2001.

\begin{tabular}{|c|c|c|c|c|}
\hline \multirow[b]{2}{*}{ Variáveis } & Daminozide & Duas semanas de DL & \multirow[b]{2}{*}{ Média } & \multirow[b]{2}{*}{$\mathrm{CV} \%{ }^{(1)}$} \\
\hline & Freqüência de Aplicação Semanal & Bissemanal & & \\
\hline Comprimento da folha $(\mathrm{cm})$ & $7,64 b^{*}$ & $8,25 \mathrm{a}$ & 7,94 & 4,28 \\
\hline Largura da folha (cm) & $4,03 \mathrm{~b}$ & $4,41 \mathrm{a}$ & 4,22 & 5,52 \\
\hline Área da folha $\left(\mathrm{cm}^{2}\right)$ & $13,75 \mathrm{~b}$ & $16,20 \mathrm{a}$ & 14,98 & 9,65 \\
\hline
\end{tabular}

* Médias não seguidas pela mesma letra na horizontal diferem entre si, pelo Teste de Duncan em nível de 5\% de probabilidade do erro.

(1) Coeficinete de variação.

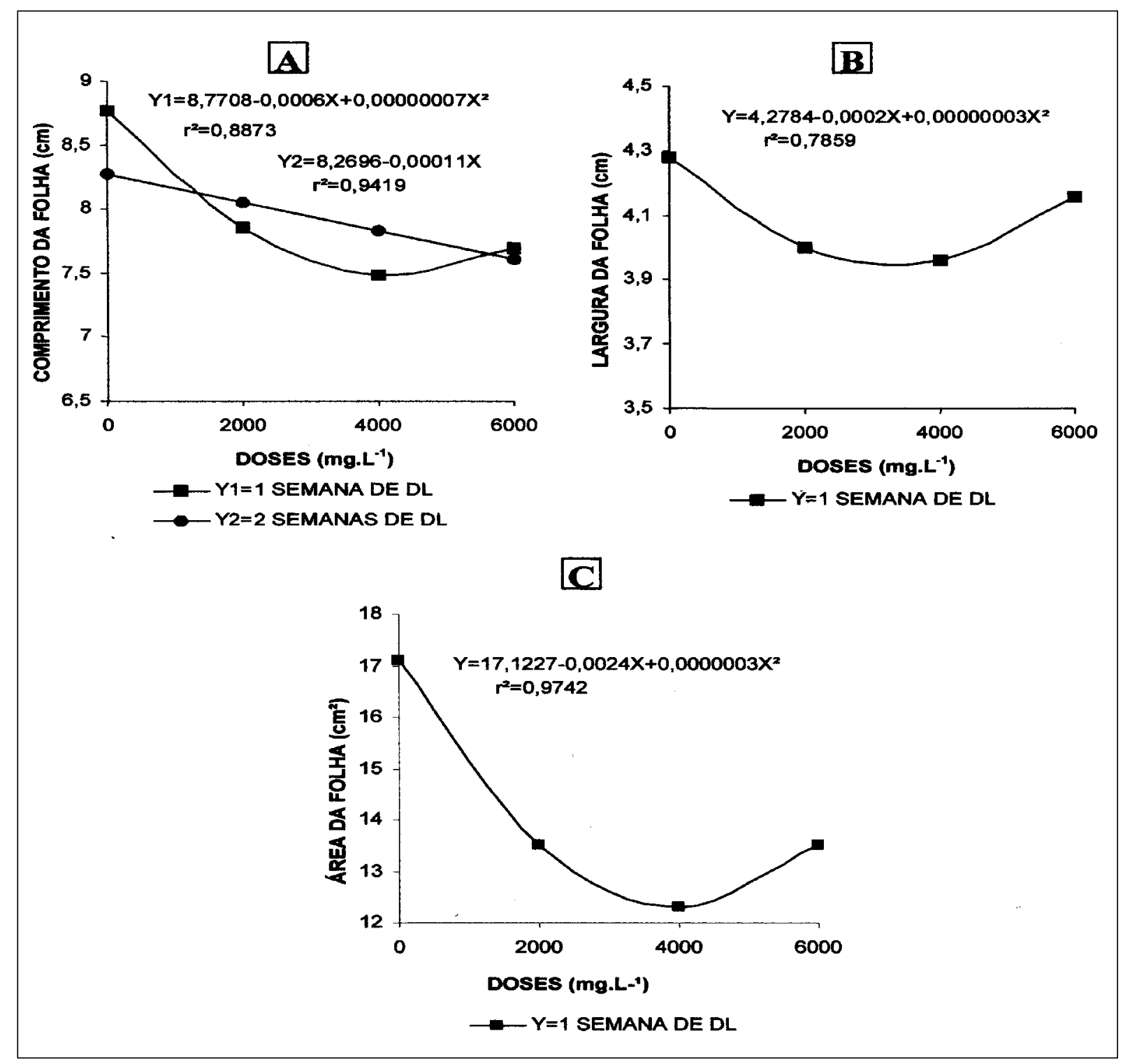

Figura 2 - Comprimento da folh (A), Largura da folha (B) e Área da folha (C) de crisântemo, cultivar Snowdon, para doses de Daminozide aplicadas nos ensaios com uma ou duas semanas de DL. Santa Maria - RS, 2001.

Ciência Rural, v.34, n.6, nov-dez, 2004. 
ensaio com uma semana de DL para a dose de $4.000 \mathrm{mg} . \mathrm{L}^{-1}$. A ação da dose sobre a largura e a área da folha foi evidenciada unicamente para o ensaio com uma semana de DL e houve diminuição das mesmas. A maior redução constatada foi também para a dose de $4.000 \mathrm{mg} . \mathrm{L}^{-1}$, na qual a largura foi reduzida em 7,50\% (Figura 2B). Na área da folha, ocorreu uma forte redução atingindo $28,03 \%$ (Figura 2C).

A redução do tamanho da folha é um grande inconveniente para o crisântemo envasado, pois as folhas grandes apresentam um papel estético importante no preenchimento dos espaços para cobertura do vaso.

A redução da área da folha também foi observada por SILVA et al. (1980) em begônia, em que o Daminozide com 2.000mg.L $\mathrm{L}^{-1}$ chegou a reduzir $76 \%$ a área foliar e por YONGKWOEN et al. (1999) em Chrysanthemum zawadskii ssp. naktongense.

\section{CONCLUSÃO}

Nas condições e época em que o trabalho foi realizado e em função dos resultados obtidos, podese concluir que a cultivar de crisântemo Snowdon conduzida em vaso e tratada com o redutor de crescimento Daminozide, apresenta prolongamento de ciclo e importante redução no tamanho de folha.

\section{REFERÊNCIAS BIBLIOGRÁFICAS}

ARTECA, R.N. Plant growth substances: principles and applications. New York: Thomson Publishing, 1995. 332p.

BARRET, J.E. Mecanisms of action. In: Tips on the use of chemical growth regulators on floriculture crops. Ohio Florists Association, p.12-18, 1992. Capturado em 23 jun. 2003. Online. Disponível na Internet http://www.uesb.br/flower/reguladores.html.

BARROS, R.S. et al. Determinação da área de folhas de café (Coffea arabica L. Bourbon Amarelo). Revista Ceres, Viçosa, v.20, n.107, p.4452, 1973.

BELLÉ, R.A. Caderno didático de floricultura. Santa Maria: RAB, 1998. 142p.

CATHEY, H.M. Comparative plant growth-retarding activities of ancymidol with ACPC, phosfon, chlormequat, and SADH on ornamental plant species. Hort Science, v.10, n.3, p.204-216, 1975.

EL-KELTAWI, N.E.; MOUSA, G.T.; MAKARY, B.S. Regulation of chrysanthemum growth using $\mathrm{GA}_{3}$ and Alar to overcome salinity depressions. In: International symposium on medicinal and aromatic plants. Acta Horticulturae, n.426, p.657-669, 1996.

HALLAIRE, M.M.; BRICHAMBAUT, M.C.P.; GOILLT, M.C. Techique d'etude des facteurs physiques de la biosphere. Paris: ENRA, 1970. 543p.

HARTLEY, D.E.; WILFRET, G.J. Poinsettias (potted) In: Tips on the use of chemical growth regulators on floriculture crops. Ohio Florists Association, p.75-77, 1992. Capturado em 23 jun. 2003. Online. Disponível na Internet http://www.uesb.br/flower/ reguladores.html.

HARTMANN, H.T. et al. Plant Science: growth, development and utilization of cultivated plants. 2.ed. New Jersey : Regents/Prentice Hall, 1988. Capturado em 23 jun. 2003. Online. Disponível na Internet http:// www.uesb.br/flower/reguladores.html.

HERTWIG, K.V. Manual de herbicidas desfolhantes, dessecantes e fitorreguladores. São Paulo : Agronômica Ceres, 1977. Capturado em 23 jun. 2003. Online. Disponível na Internet http://www.uesb.br/flower/reguladores.html.

KOFRANEK, A.M. Cut chrysanthemum. In: LARSON, A.R. Introduction to floriculture. 2.ed. New York : Academic, 1992. p.3-42.

LOPES, L.C. O cultivo do crisântemo. Viçosa : Universidade Federal Viçosa, 1977. 12p. (Boletim de extensão, 22).

LOZOYA-SALDAÑA, H. Inhibidores de crescimento para margarita (Chrysanthemum morifolium Ramat) em maceta. I: alar y cycocel. Revista Chapingo, Série Horticultura, México, n.78, p.19-24, 1992.

MOTOS, J. Produção de crisântemos em vaso. Holambra : Flortec, 1998. 34p.

NARDI, C. Rendimento e qualidade do crisântemo (Dendranthema grandiflora Tzvelev.) cultivar Snowdon em diferentes populações e épocas de plantio. 2000 . $73 \mathrm{f}$. Dissertação (Mestrado em Agronomia) - Curso de Pós-graduação em Agronomia, Universidade Federal de Santa Maria.

PENON, P. Médiateurs moléculaires de la croissance et du développement. In: MAZLIAK, P. Croissance et développement. Physiologie végétale II. Paris : Hermann Collection Méthodes, 1982. Cap.1, p.1-45.

SCHOENMAKER-VAN ZANTEN. Catálogo composto. São Paulo, 1999-00. 21p.

SILVA, M.P.F. et al. Uso do Alar no controle do crescimento de Begônia FLORICULTURA E PLANTAS ORNAMENTAIS,1., 1980, Campinas. Resumos... Capturado em 23 jun. 2003. Online. Disponível na Internet http://www.uesb.br/flower/reguladores.html.

TAYAMA, H.K. Chrysanthemums (Potted). In: Tips on the use of chemical growth regulators on floriculture crops. Ohio Florists Association, p.40-41, 1992. Capturado em 23 jun. 2003. Online. Disponível na Internet http://www.uesb.br/flower/reguladores.html.

WEI, S.; BIWEN, H. Studies on production of desk chrysanthemum applying $B_{9}$ as dwarfing agent. Journal of China Agricultural University, v.2, n.3, p.101-105, 1997. (Resumo publicado no Horticultural Abstracts, v.68, n.9, p.1048, 1998).

YONGKWEON, Y.; SANGWOOK, K.; HYUNKYUNG, K. Effects of pinching and daminozide treatment on the growth and flowering of Chrysanthemum zawadskii ssp. naktongense. Journal of the Korean Society for Horticultural Science, v.40, n.5, p.598-602, 1999. (Resumo publicado no Horticultural Abstracts, v. 70, n.4, p. 449, 2000). 